\title{
EFEITOS DIRETOS E INDIRETOS E CORRELAÇÕES CANÔNICAS PARA CARACTERES RELACIONADOS COM A PRODUÇÃO DE PIMENTÃO ${ }^{(1)}$
}

\author{
MARCELO TAVARES ${ }^{(2)}$, ARLETE MARCHI TAVARES DE MELO ${ }^{(3)}$ \\ \& WALKYRIA BUENO SCIVITTARO ${ }^{(4)}$
}

\begin{abstract}
RESUMO
O trabalho foi realizado com os seguintes objetivos: (a) estudar os efeitos diretos e indiretos de oito caracteres de planta e fruto sobre a produção de pimentão (Capsicum annuum L.), utilizando-se de análise de trilha, e (b) obter as correlações canônicas entre o grupo dos principais fatores de produção (peso ${ }^{(5)}$ e número total de frutos) e os grupos: (1) produção precoce-número de frutos e produção precoce-peso de frutos; (2) comprimento, largura e número de lóculos do fruto, e (3) comprimento e largura do fruto. Empregaram-se seis genótipos de pimentão e os híbridos possíveis entre eles, obtidos mediante esquema dialélico, excluindo-se os recíprocos. Os resultados mostraram que as avaliações da produção de pimentão podem ser feitas nas primeiras colheitas, em vista do efeito pronunciado do caráter produção precoce-peso de frutos sobre o peso total de frutos, e da significância das correlações canônicas entre produção precoce-número e peso de frutos e peso total de frutos. O número total de frutos foi o caráter que apresentou maior efeito positivo sobre o peso total de frutos. Menor largura e maior número de lóculos por fruto determinaram maior peso total de frutos.
\end{abstract}

Termos de indexação: análise do coeficiente de trilha, correlação canônica, pimentão, Capsicum annuum $\mathrm{L}$.

\footnotetext{
(1) Recebido para publicação em 6 de fevereiro de 1998 e aceito em 5 de abril de 1999.

(2) Departamento de Matemática da Universidade Federal de Uberlândia (UFU), Avenida João Naves de Ávila, 2160, Câmpus Santa Mônica, 34408-100 Uberlândia (MG).

(3) Centro de Horticultura, Instituto Agronômico (IAC), Caixa Postal 28, 13001-970 Campinas (SP).

(4) Centro de Pesquisa Agropecuário de Clima Temperado (CPACT-EMBRAPA), Caixa Postal 403, 96001-970 Pelotas (RS).

(5) Peso: refere-se à massa, em gramas, de acordo com o Sistema Internacional de Unidades.
} 


\title{
ABSTRACT \\ DIRECT AND INDIRECT EFFECTS AND CANONICAL CORRELATIONS OF AGRONOMIC TRAITS ON SWEET PEPPER YIELD
}

\begin{abstract}
A study was carried out to evaluate: (a) direct and indirect effects (path analysis) of eight plant and fruit traits on sweet pepper (Capsicum annuum L.) yield; and (b) canonical correlations among the main production factors group (fruit weight and fruit number) and the following groups: (i) early yield (fruit weight and fruit number); (ii) fruit length, fruit width and fruit loculi number; and (iii) fruit length and fruit width. It was studied six parental genotypes, and the $\mathrm{F}_{1}$ hybrids among excluding the reciprocals. The results showed that yield evaluations might be done at the beginning of the harvest due to the remarkable effects of early yields on total fruit weigth, and the canonical correlations significance between early yield and total fruit weigth. Total fruit number was the variable which showed greater positive effect on total fruit weigth. Reduced fruit width and large number of fruit loculi are correlated with increased total fruit weigth.
\end{abstract}

Index terms: path analysis, canonical correlations, sweet pepper, Capsicum annuum L.

\section{INTRODUÇÃO}

Nos programas de melhoramento, é necessário que a seleção de genótipos superiores seja feita da maneira mais eficiente possível. Nas diversas culturas, essa superioridade se dá, normalmente, mediante a variável produção, que é resultado da interação de fatores genéticos, fisiológicos e ambientais. A complexidade desses fatores e a necessidade de maior eficiência no processo de seleção requerem o desdobramento das estimativas de correlação genética. Tal desdobramento pode ser realizado pela análise de trilha, desenvolvida por Wrigth $(1921,1923)$ e detalhada por Li (1975): consiste no estudo dos efeitos diretos e indiretos de caracteres sobre uma variável básica. Apesar de sua grande importância, a análise de trilha possui uma limitação, por se utilizar apenas de uma variável dependente. Para contornar o problema, pode-se fazer uso conjunto de outro tipo de análise: as correlações canônicas, as quais permitem conhecer, de maneira mais detalhada, as inter-relações entre os vários caracteres usados durante o processo de seleção.
O conhecimento das estimativas de correlação pode contribuir com o trabalho do melhorista quando associado a duas novas estimativas, a saber: a resposta correlacionada à seleção e componentes da interação genótipos por ambiente.

Relações entre caracteres da planta e produção podem ser estudadas para prever o comportamento dos genótipos, possibilitando a redução do número de ensaios de competição, bastante dispendiosos (Hallauer \& Miranda Filho, 1981).

Em pimentão, no estudo de correlações, a maioria dos trabalhos encontrados na literatura dá ênfase à correlação entre a produção e outros caracteres que a influenciam de maneira mais acentuada. A produção depende, pois, fundamentalmente, do peso ${ }^{(5)} \mathrm{e}$ do número de frutos por planta (Rocchetta et al., 1976; Braz, 1982; Gupta \& Yadav, 1984; Anand et al., 1987; Deprestre et al., 1988; Ghai \& Thakur, 1989; Melo, 1997). Para Silvetti (1991), um programa de seleção que pretenda elevar a produção por planta, deveria basear-se no aumento do número ou peso ${ }^{(5)}$ de frutos

\footnotetext{
(5) Peso: refere-se à massa, em gramas, de acordo com o Sistema Internacional de Unidades.
} 
por planta. Por sua vez, Deprestre et al. (1988) mostraram que a largura do fruto apresenta efeito direto sobre a produção.

A análise de trilha realizada por Cruz et al. (1988) revelou que o tamanho do fruto de pimentão, expresso em termos de comprimento e largura, tem efeito direto e positivo sobre a produção total de frutos nas populações parentais e nos híbridos. Os caracteres número total de frutos, dias para o florescimento e altura da planta têm influência distinta sobre a produção total de frutos, pois apresentam efeitos positivos na população parental e negativos na híbrida.

Este trabalho teve por objetivos: (a) estudar, por meio da análise de trilha, os efeitos diretos e indiretos de oito caracteres de planta e fruto sobre a produção de pimentão, e (b) obter as correlações canônicas a partir do grupo dos principais fatores da produção (peso e número total de frutos) e dos caracteres de fruto.

\section{MATERIAL E MÉTODOS}

No experimento, realizado na Escola Superior de Agricultura de Lavras (ESAL), em Lavras (MG) utilizaram-se seis genótipos de pimentão escolhidos por suas características e/ou origem: (1) Linha 4; (2) Linha 6; (3) Linha 8; (4) Agronômico 8; (5) Ikeda, e (6) Magda e os híbridos possíveis entre eles, excluindo-se os recíprocos.

Os tratamentos, compreendendo quinze híbridos e seis parentais, foram dispostos no delineamento de blocos casualizados, com três repetições. A parcela experimental foi constituída por uma fileira de 7,5 m de comprimento com quinze plantas, sendo empregadas, para a coleta de dados, as cinco mais representativas, ou seja, as que se mostraram mais competitivas dentro da parcela. Mais detalhes experimentais encontram-se em Tavares (1993).
As correlações apresentadas por Tavares (1993) foram desdobradas, mediante a análise de trilha (Wrigth, 1921, 1923; Li, 1975), em efeitos diretos e indiretos, nas populações híbrida e parental em conjunto. O peso total de frutos por planta (PTF) foi considerado como variável dependente e, como variáveis explicativas, os seguintes caracteres: número total de frutos (NTF), produção precoce-número de frutos (PPNF), produção precoce-peso de frutos (PPPF), peso médio do fruto (PMF), comprimento (COMP), largura (LARG) e número de lóculos do fruto (LOC) e altura da planta (ALT). As produções precoces, o número e o peso de frutos, correspondem às quatro primeiras colheitas.

Foram obtidas, ainda, estimativas das correlações canônicas (Cruz \& Regazzi, 1994) do grupo composto pelo peso total e número total de frutos, com os seguintes grupos: (1) produção precoce-número de frutos e produção precoce-peso de frutos; (2) comprimento, largura e número de lóculos do fruto; e (3) comprimento e largura do fruto.

\section{RESULTADOS E DISCUSSÃO}

O coeficiente de determinação obtido para a análise de trilha foi de 0,8862 e a magnitude do efeito da variável residual, de 0,3372 .

O número total de frutos apresentou o maior efeito direto em relação ao peso total de frutos, estimado em 1,2626. Esse valor foi 3,74 vezes maior que o da variável residual $(0,3372)$. A estimativa do efeito direto do peso médio de frutos $(0,6173)$ também superou o valor do efeito da variável residual, apesar de não apresentar uma magnitude tão elevada. Também o peso médio de frutos revelou efeito negativo, via número total de frutos, de igual magnitude, diminuindo, assim, sua importância. Apenas produção precoce-número de frutos, comprimento e número de lóculos do fruto apresentaram estimativas negativas em seus efeitos diretos (Quadro 1). 
Quadro 1. Efeitos direto, indireto e total de oito caracteres agronômicos sobre o peso total de frutos (PTF) de pimentão

\begin{tabular}{|c|c|c|c|c|c|c|c|}
\hline Caráter $^{(1)}$ & Efeito & Via & PTF & Caráter $^{(1)}$ & Efeito & Via & PTF \\
\hline \multirow[t]{9}{*}{ NTF } & Direto & & 1,2626 & \multirow[t]{9}{*}{ COMP } & Direto & & $-0,1611$ \\
\hline & \multirow[t]{7}{*}{ Indireto } & PPNF & $-0,2633$ & & \multirow[t]{7}{*}{ Indireto } & NTF & 0,2032 \\
\hline & & PPPF & 0,0533 & & & PPNF & 0,0913 \\
\hline & & ALT & 0,0355 & & & PPPF & $-0,1797$ \\
\hline & & COMP & $-0,0259$ & & & ALT & 0,1022 \\
\hline & & LARG & $-0,0259$ & & & LARG & 0,0382 \\
\hline & & \multirow{3}{*}{$\begin{array}{l}\text { LOC } \\
\text { PMF }\end{array}$} & 0,0013 & & & LOC & $-0,0030$ \\
\hline & & & $-0,3136$ & & & \multirow[t]{2}{*}{ PMF } & $-0,2771$ \\
\hline & Total & & 0,7240 & & Total & & $-0,1860$ \\
\hline \multirow[t]{9}{*}{ PPNF } & \multirow{8}{*}{$\begin{array}{l}\text { Direto } \\
\text { Indireto }\end{array}$} & & $-0,3553$ & \multirow[t]{9}{*}{ LARG } & Direto & & 0,0711 \\
\hline & & NTF & 0,9356 & & \multirow[t]{7}{*}{ Indireto } & NTF & $-0,4608$ \\
\hline & & PPPF & 0,1491 & & & PPNF & $-0,3468$ \\
\hline & & ALT & 0,0254 & & & PPPF & $-0,0297$ \\
\hline & & COMP & 0,0414 & & & ALT & $-0,2142$ \\
\hline & & LARG & 0,0694 & & & COMP & $-0,0865$ \\
\hline & & LOC & 0,0066 & & & LOC & $-0,0140$ \\
\hline & & PMF & 0,0555 & & & PMF & 0,5790 \\
\hline & Total & & 0,9280 & & \multicolumn{2}{|l|}{ Total } & $-0,5020$ \\
\hline \multirow[t]{9}{*}{ PPPF } & Direto & & 0,1970 & \multirow[t]{9}{*}{ LOC } & Direto & & $-0,0213$ \\
\hline & \multirow[t]{7}{*}{ Indireto } & NTF & 0,3421 & & \multirow[t]{7}{*}{ Indireto } & NTF & $-0,0770$ \\
\hline & & PPPF & $-0,2690$ & & & PPNF & 0,1112 \\
\hline & & ALT & $-0,1386$ & & & PPPF & $-0,0047$ \\
\hline & & COMP & 0,1469 & & & ALT & $-0,0079$ \\
\hline & & LARG & $-0,0107$ & & & COMP & $-0,0228$ \\
\hline & & LOC & 0,0005 & & & LARG & 0,0467 \\
\hline & & PMF & 0,5667 & & & PMF & 0,1839 \\
\hline & Total & & 0,8350 & & Total & & 0,2080 \\
\hline \multirow[t]{8}{*}{ PMF } & Direto & & 0,6173 & ALT & \multirow{9}{*}{$\begin{array}{l}\text { Direto } \\
\text { Indireto }\end{array}$} & & 0,2276 \\
\hline & Indireto & NTF & $-0,6414$ & & & NTF & 0,1969 \\
\hline & & PPNF & $-0,0319$ & & & PPNF & $-0,0398$ \\
\hline & & PPPF & 0,1808 & & & PPPF & $-0,1200$ \\
\hline & & ALT & $-0,0325$ & & & COMP & $-0,0723$ \\
\hline & & COMP & 0,0723 & & & LARG & $-0,0669$ \\
\hline & & LARG & 0,0667 & & & LOC & 0,0007 \\
\hline & & LOC & $-0,0063$ & \multirow[b]{2}{*}{ Total } & & PMF & $-0,0882$ \\
\hline To & & & 0,2250 & & & & 0,0380 \\
\hline
\end{tabular}

(1) NTF: número total de frutos; PPNF: produção precoce-número de frutos; PPPF: produção precoce-peso de frutos; PMF: peso médio de frutos. COMP: comprimento de frutos; LARG: largura de frutos; LOC: número de lóculos do fruto; ALT: altura de planta. 
Os caracteres número total de frutos, produção precoce-peso de frutos, peso médio do fruto, comprimento do fruto e altura da planta apresentaram estimativas de efeito direto com o mesmo sinal das correlações genotípicas obtidas por Tavares (1993) (Quadros 1 e 3).

Considerando o efeito total, os caracteres que apresentaram maior efeito sobre o peso total de frutos foram os seguintes: número total de frutos, produção precoce-número de frutos e produção precoce-peso de frutos, cujos valores foram superiores a 0,7 (Quadro1) e apresentaram uma correlação genotípica significativa para esses caracteres (Quadro 3).

Verifica-se - Quadro 1 - que o efeito direto do peso médio de frutos sobre o peso total de frutos $(0,6173)$, em relação à magnitude do efeito da variância residual $(0,3372)$, foi de 1,83 , sendo semelhante aos resultados encontrados por Sahoo et al. (1990). A correlação genotípica, porém, entre esses dois caracteres, não foi significativa, como se pode ver no Quadro 3. Esses resultados sugerem que o uso dessa variável auxiliar não deve ser descartado em seleções indiretas, pois a seleção simultânea para vários caracteres pode apresentar bons resultados, tomando cuidado apenas para a variável número total de frutos, com um valor maior no efeito indireto $(-0,6414)$, no sentido de diminuir o peso total de frutos, já que maior número de frutos por planta resulta em maior competição entre frutos e, conseqüentemente, frutos de menor peso.

A produção precoce-número de frutos foi o único caráter com elevada estimativa de efeito indireto sobre o peso total de frutos $(0,9356)$, sendo esse efeito obtido via número total de frutos (Quadro 1), mostrando, assim, a importância desse caráter na obtenção de plantas com maior peso de frutos por planta.

As correlações canônicas entre o grupo peso total de frutos e número total de frutos com o grupo produção precoce-número de frutos e produção precoce-peso de frutos foram de alta magnitude e significativas para os dois pares canônicos (Quadro 2).
Quadro 2. Correlações canônicas e pares canônicos estimados entre os componentes primários da produção com grupos de produção precoce e características de fruto

\begin{tabular}{|c|c|c|}
\hline \multirow{2}{*}{ Característica $^{(1)}$} & \multicolumn{2}{|c|}{ Par canônico } \\
\hline & Primeiro & Segundo \\
\hline PTF ......................... & 1,3270 & $-0,5837$ \\
\hline NTF ..................... & $-0,5579$ & 1,3379 \\
\hline PPNF ……............... & 0,2195 & 1,2746 \\
\hline PPPF .................... & 0,7909 & $-1,0897$ \\
\hline r & 0,9675 & 0,8421 \\
\hline Significância .. & $* *$ & $* *$ \\
\hline PTF ........................ & 1,2199 & $-0,7832$ \\
\hline NTF ........................ & $-0,3427$ & 1,4084 \\
\hline COMP .................. & 0,3161 & 0,5913 \\
\hline 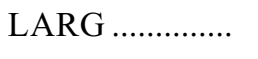 & $-1,4216$ & $-0,3867$ \\
\hline 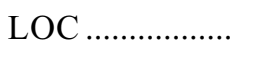 & 1,1638 & $-0,0788$ \\
\hline r & 0,9608 & 0,5353 \\
\hline Significância .. & $* *$ & n.s. \\
\hline PTF ........................ & $-0,0187$ & 1,4496 \\
\hline 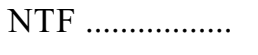 & 1,0134 & $-1,0364$ \\
\hline COMP ................. & 0,5062 & $-0,3497$ \\
\hline 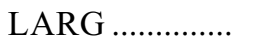 & $-0,6324$ & $-0,1616$ \\
\hline r & 0,5588 & 0,4572 \\
\hline Significância .. & n.s. & n.s. \\
\hline
\end{tabular}

(1) PTF: peso total de frutos; NTF:número total de frutos; PPNF: produção precoce-número de frutos; PPPF: produção precoce-peso de frutos. COMP: comprimento de frutos; LARG: largura de frutos; LOC: número de lóculos do fruto; PMF: peso médio de frutos; ALT: altura de planta. 
Quadro 3. Estimativa dos coeficientes de correlação genotípica $\left(\mathrm{r}_{\mathrm{G}}\right)$ entre os caracteres estudados ${ }^{(1)}$ (Adaptada de TAVARES, 1993)

\begin{tabular}{lcccccccc}
\hline Caracteres & NTF & PPNF & PPPF & ALT & COMP & LARG & LOC & PMF \\
\hline PTF ...... & $0,724^{* *}$ & $0,928^{* *}$ & $0,835^{* *}$ & 0,038 & $-0,186$ & $-0,502$ & 0,208 & 0,225 \\
NTF ..... & - & $0,741^{* *}$ & 0,271 & 0,156 & 0,161 & $-0,365$ & $-0,061$ & $-0,939^{* *}$ \\
PPNF .... & - & - & $0,757^{* *}$ & 0,112 & $-0,257$ & $0,976^{* *}$ & $-0,313$ & 0,090 \\
PPPF .... & - & - & - & $-0,609^{* *}$ & $-0,912^{* *}$ & $-0,151$ & $-0,024$ & $0,918^{* *}$ \\
ALT ...... & - & - & - & - & $0,449^{*}$ & $0,941^{* *}$ & $-0,035$ & $-0,143$ \\
COMP .. & - & - & - & - & - & $0,537^{*}$ & 0,142 & $-0,449^{*}$ \\
LARG ... & - & - & - & - & - & - & $0,657^{* *}$ & $0,938^{* *}$ \\
LOC ...... & - & - & - & - & - & - & - & 0,298 \\
\hline
\end{tabular}

(1) PTF: peso total de frutos; NTF:número total de frutos; PPNF: produção precoce-número de frutos; PPPF: produção precoce-peso de frutos; COMP: comprimento de frutos; LARG: largura de frutos; LOC: número de lóculos do fruto; PMF: peso médio de frutos; ALT: altura de planta.

Isso evidencia que a seleção de genótipos superiores pode ser realizada nas primeiras colheitas, eliminando a necessidade de um longo período de colheita. Com isso, haveria diminuição e conseqüente otimização dos custos de um programa de melhoramento. Verifica-se a ocorrência de sinais negativos nos pares canônicos estimados, em relação às produções precoces, já que o número e o peso de frutos se correlacionam negativamente.

Para os demais grupos de correlação (Quadro 2), não houve significância, exceto para o primeiro par canônico formado pelos grupos: peso e número total de frutos, e comprimento, largura e número de lóculos do fruto. Esses resultados sugerem que o peso total de frutos será maior em plantas que produzam frutos com menor largura e maior número de lóculos. Isso pode ser explicado pelo fato de os frutos de menor largura apresentarem maior desenvolvimento no comprimento ou maior espessura de polpa.

\section{CONCLUSÕES}

1. As avaliações da produção de pimentão podem ser feitas nas primeiras colheitas, em função do efeito pronunciado de produção precoce-peso de frutos sobre a produção total de frutos e da significância das correlações canônicas entre produção total de frutos com produção precoce-número de frutos e produção precoce-peso de frutos.

2. O número total de frutos foi o caráter com maior efeito positivo sobre o peso total de frutos.

3. Menor largura e maior número de lóculos do fruto determinaram maior peso total de frutos.

\section{REFERÊNCIAS BIBLIOGRÁFICAS}

ANAND, N.; DESHPANDE, A.A. \& RAMACHANDER, P.R. Intra-group geometry in Capsicum annuum L. Genetica Agraria, Roma, 41:453-460, 1987.

BRAZ, L.T. Avaliação de caracteres agronômicos e qualitativos de três cultivares de pimentão (Capsicum annuum L.) e da heterose em seus híbridos $\mathrm{F}_{1}$. Viçosa, 1982. 75p. Dissertação (Mestrado). Universidade Federal de Viçosa, 1982.

CRUZ, C.D.; MIRANDA, J.E.C. de \& COSTA, C.P. da. Correlações, efeitos diretos e indiretos de caracteres agronômicos sobre a produção de pimentão (Capsicum annuum L.). Revista Brasileira de Genética, Ribeirão Preto, 11(4):921-918, 1988. 
CRUZ, C.D. \& REGAZZI, A. J. Modelos biométricos aplicados no melhoramento genético. Viçosa, UFV, Imprensa Universitária, 1994. 390p.

DEPESTRE, T.; GOMEZ, O. \& ESPINOSA, J. Path coefficient analysis in sweet pepper. Capsicum Newsletter, Turin, 7:37-38, 1988 .

GHAI, T.R. \& THAKUR, M.R. Variability and correlation studies in an intervarietal cross of chilli. Punjab Horticultural Journal, Ludhiana 27(1/2):80-83, 1987. Plant Breeding Abstracts, Cambridge, 59(8):775, 1989. (Resumo 7077)

GUPTA, C.T. \& YADAV, R.D.S. Genetic variability and path analysis in chilli (Capsicum annuum L.). Genetica Agraria, Roma, 38(4):425-432, 1984.

HALLAUER, A.R. \& MIRANDA FILHO, J.B. Quantitative genetics in maize breeding. Ames, Iowa State University Press, 1981. 468p.

LI, C.C. Path analysis: a primer. Boxwood, Pacific Grove, 1975. 346p.

MELO, A.M.T. Análise genética de caracteres de fruto em hibridos de pimentão. Piracicaba, 1997. 112p. Tese (Doutorado) - Escola Superior de Agricultura "Luiz de Queiroz". 1997
ROCCHETTA, G.; GIORGI, G. \& GIOVANNELLI, G. Correlation analysis between morphological traits and productivity in cultivate Capsicum for an understanding of the heterosis phenomenon. Genetica Agraria, Roma, 30(3):355-374, 1976.

SAHOO, S. C.; MISHRA, S. N. \& MISHRA, R. S. Association of traits in segregating populacion of chilli (Capsicum annuum L.). Indian Journal of Agricultural Sciences. New Delhi, 60 (4): 233-236, 1990.

SILVETTI, E. Selection for yield correlated responses in Capsicum. In: EUCARPIA MEETING ON GENETICS AND BREEDING OF CAPSICUM AND EGGPLANT, 7., Kragujevac, 1989. Proceedings. Kragujevac, EUCARPIA, 1989. s.p. Plant Breeding Abstracts, Cambridge, 61(2):234, 1991. (Resumo 1877)

TAVARES, M. Heterose e estimativa de parâmetros genéticos em um cruzamento dialélico de pimentão (Capsicum annuит L.). Lavras, 1993. 88p. Dissertação (Mestrado) - Escola Superior de Agricultura de Lavras, 1993.

WRIGHT, S. Correlation and causation. Journal of Agricultural Research, Washington, 20:557-585, 1921.

WRIGHT, S. The theory of path coefficients - a replay to Nile's criticism. Genetics, Austin, 8:239-255, 1923. 